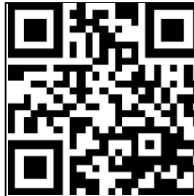

Editor's choice

can to access mo free content

\title{
Impact of clinical pharmacist intervention on length of stay in an acute admission unit: a cluster randomised study
}

\author{
Katrine Brodersen Lind, ${ }^{1}$ Charlotte Arp Soerensen, ${ }^{2}$ Suheil Andreas Salamon, ${ }^{3}$ \\ Tommy Midtgaard Jensen, ${ }^{1}$ Hans Kirkegaard, ${ }^{4}$ Marianne Lisby ${ }^{4}$
}

\begin{abstract}
${ }^{1}$ Emergency Department, Randers Regional Hospital, Randers, Denmark

${ }^{2}$ Randers Department, Hospital Pharmacy Central Denmark Region, Randers, Denmark ${ }^{3}$ Department of Emergency Medicine, Aalborg University Hospital, Aalborg, Denmark

${ }^{4}$ Research Center for Emergency Medicine, Aarhus University Hospital, Aarhus, Denmark
\end{abstract}

\section{Correspondence to} Katrine Brodersen Lind, Emergency Department, Randers Regional Hospital, Viborgvej 32, 2. Tv, Aarhus C, Randers 8000, Denmark; katrine.brodersen@gmail.com

Received 4 August 2015 Revised 21 October 2015 Accepted 2 November 2015 Published Online First 1 December 2015

\begin{abstract}
Objectives Physicians in acute admission units (AAUs) are obliged to obtain medication history and perform medication reconciliation, which is time consuming and often incomplete. Studies show that clinical pharmacists (CPs) can obtain accurate medication histories, but so far no studies have investigated the effect of this on time measures. Therefore, the objective of the present study was to investigate the effect of a CP intervention on length of stay (LOS) in an AAU.
\end{abstract}

Methods The study was designed as a prospective, cluster randomised study. Weekdays were randomised to control or intervention. CP intervention consisted of obtaining medication history and performing medication reconciliation and review. The primary outcome was LOS in the AAU. Secondary outcomes were other timerelated measures-for example, physicians' self-reported time spent on medication topics. Finally, the number of documented medications per patient was established.

Results 232 and 216 patients, respectively, were included on control $(n=63)$ and intervention $(n=63)$ days. The mean LOS was $342(95 \% \mathrm{Cl} 323$ to 362) $\mathrm{min}$ in the intervention group and $339(95 \% \mathrm{Cl}$ 322 to 357) min in the control group, which was not statistically significantly different. Physicians spent on average $4.3(95 \% \mathrm{Cl} 3.7$ to 5.0$) \mathrm{min}$ in the intervention group and $7.5(95 \% \mathrm{Cl} 6.6$ to 8.5$) \mathrm{min}$ in the control group, corresponding to an overall reduction of $43.0 \%(95 \% \mathrm{Cl} 30.9 \%$ to $53.0 \%$, $\mathrm{p}<0.001)$. The number of documented medications per patient was 10.0 (intervention group) and 8.8 (control group).

Conclusions This study indicates that LOS in the AAU was not affected by CP intervention; however, physicians reported a significant reduction in time spent on medication topics.

Trial registration number Clinical Trial Gov: 1-16-02-379-13.

\section{INTRODUCTION}

Quality and efficiency in the emergency department (ED) concerns clinicians and administrators worldwide. EDs are challenged by increasing numbers of patients and a desire to optimise flow, avoid crowding, and increase the quality of treatment. Crowding delays treatment and increases the length of stay (LOS) in the ED, even among high-acuity patients. ${ }^{12}$ Periods of high ED crowding, and thereby prolonged stay in the ED, are associated with increased inpatient mortality and adverse events in older patients. $^{13}$
In Denmark, many EDs are divided into an emergency unit (EU)-taking care of traumas and injuries - and an observational unit, often named the acute admission unit (AAU) - to which medical and surgical patients who require additional observation before a decision is made about hospitalisation or discharge can be referred. Physicians have to obtain a medication history and perform medication reconciliation for patients referred to the AAU. These tasks are time consuming, and studies have shown that medication histories obtained by physicians are often incomplete. ${ }^{4-6}$

Errors in physician-obtained medication histories occur in up to $67 \%$ of cases compared with comprehensive medication histories. ${ }^{6}$ Various studies have shown that clinical pharmacists (CPs) can be used in the ED to obtain accurate medication histories. $^{5}{ }^{7-9}$ If CPs are used to obtain medication histories, presumably physicians will save time and thereby decrease LOS and crowding in the AAU.

The aim of this study was to investigate the effect of a CP intervention on patient LOS and other time measures in an AAU.

\section{METHODS}

\section{Trial design}

The study was designed as a prospective, cluster randomised study. Weekdays were randomised to either control or intervention. Cluster randomisation was chosen in order to avoid any possible contamination between study groups in the primary outcome measure, otherwise physician time saved on intervention patients could potentially lead to shorter LOS for control patients because of improved flow.

\section{Participants}

The clusters consisted of patients arriving at the AAU at Randers Regional Hospital, Denmark, from 22 October 2013 until 1 May 2014 on weekdays from 09:00 to 16:15. Weekends and holidays were excluded as clusters.

Patients aged $\geq 18$ years, taking $\geq 4$ drugs daily (including over-the-counter drugs, herbals and supplements) were eligible for inclusion. Terminal or intoxicated patients, patients assigned to triage level 1, patients referred to the acute outpatient clinic, and patients unable to give informed consent were not eligible for inclusion. In addition, patients could not be included if the physician had interviewed them before written informed consent had been obtained or if it was not possible to include them before 16:30. Patients who unexpectedly spent the night in the AAU were also excluded to 
avoid possible interference with the primary outcome caused by artificial prolongation of LOS (see figure 2).

The Regional Committee of Health Care Ethics waived the need for approval. The study was registered in the Danish Data Protection Agency and ClinicalTrials.gov (ID-number: 1-16-02-379-13).

\section{Randomisation}

Randomisation was performed by the hospital pharmacy's department of quality assurance by using http://www. randomization.com and block sizes from 8 to 18 to avoid possible prediction of the distribution. For each cluster, the allocations were written down and placed in a sealed opaque envelope.

Each morning, the AAU staff were informed whether the day was allocated to control or intervention.

\section{Study setting}

The ED at Randers Regional Hospital has an annual intake of around 32000 patients/year. Approximately 9000 of these patients are referred to the AAU, which receives medical and surgical patients (except cardiology, gynaecology, paediatric and orthopaedic patients).

\section{Control/standard care}

On arrival, patients were triaged on a scale from level 1 to 4 by a nurse (using a modified Danish version of the ABCDE algorithm). ${ }^{10}$ After triage, they were seen by a physician.

Besides examination, the physician was responsible for obtaining a medication history, reconciling and assessing overall medication treatment, and entering approved prescriptions into the electronic medication module (EMM).

\section{Interventions}

Two CPs from the hospital pharmacy carried out the interventions. The CP intervention consisted of obtaining the medication history, entering prescriptions into the EMM, medication reconciliation, reviewing the overall medication treatment, and writing a CP note in the electronic medical record (EMR). The $\mathrm{CP}$ intervention replaced the physician's tasks related to medication apart from assessing and approving the suggested prescriptions in the EMM.

The CP had to use a minimum of two sources for information about the medication-for example, electronic shared medication record, ${ }^{11}$ medication list, or phone calls to the patient's general practitioner. One source was, when possible, an interview with the patient, relatives or both. The interview was performed directly after triage and before the physician examined the patient. While the physician examined the patient, the CP completed the intervention (figure 1).
Medication reconciliation involved compiling all available information about the medication history and comparing this with the prescriptions in the EMM.

The medication review comprised an assessment of indications, contraindications, dosages, effects, interactions, availability and costs of each prescription. Proposed changes in therapy were communicated to the physician by the CP as a note in the EMR. The CP had permission to perform changes in time of administration due to pharmacological issues (eg, interactions, side effects, absorption) and generic replacement with cheaper medications as required by Danish law.

\section{Outcomes}

The primary outcome measure was LOS in the AAU, defined as the interval in minutes between arrival and discharge or transfer to a hospital ward as registered in the EMR by secretaries.

The secondary outcome measures are shown in table 1 .

Time from arrival to a treatment plan (TATP) is a shorter time measure than LOS. TATP is defined as the TATP documented in the EMR. The admitting physician was responsible for the treatment plan.

\section{Sample-size calculation}

The calculation of sample size was based on a power of $85 \%$ and a statistical significance level of 5\%. The SD for LOS was 133.6 min based on results from a pilot study (unpublished). An average reduction of 30 min between study groups was considered a clinically relevant difference in LOS. This was based on data from a study with a similar CP intervention taking 44 min. ${ }^{12}$ To take clustering into account, we used an intracluster correlation coefficient (ICC) of 0.05 . We chose this estimate since most reported ICCs in studies using shifts as a clustering unit tended to be small and rarely exceeded $0.05 .{ }^{13}{ }^{14}$ On the basis of these values, we calculated a sample size of 437 persons in each of the study groups.

\section{Statistical analysis}

All data were analysed using Stata (V.13). Descriptive statistics were calculated for variables of interest using counts and percentages, unless otherwise specified, and are presented with 95\% CIs.

By nature, the distribution of all time variables is positively skewed, and therefore all time variables were log-transformed before any analysis was performed. This implies that all analyses were performed on parametric data. All time variables were summarised using means and are presented with 95\% CIs.

Comparative analyses of time variables were performed between study groups and adjusted for clustering by using robust SEs. Time variables were tested by linear regression in order to adjust for clustering. LOS and TATP were also adjusted for medical specialty.
Figure 1 Flow of the patient's stay in the acute admission unit. The physician's duties regarding medication treatment (dotted area) were performed by the clinical pharmacist (CP) in the intervention group. LOS, length of stay.

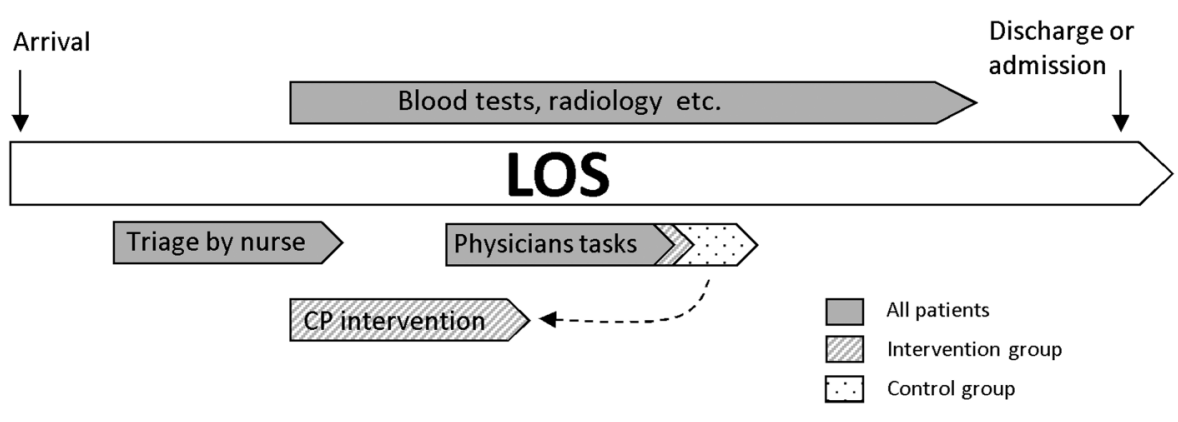

Lind KB, et al. Eur J Hosp Pharm 2016;23:171-176. doi:10.1136/ejhpharm-2015-000767 


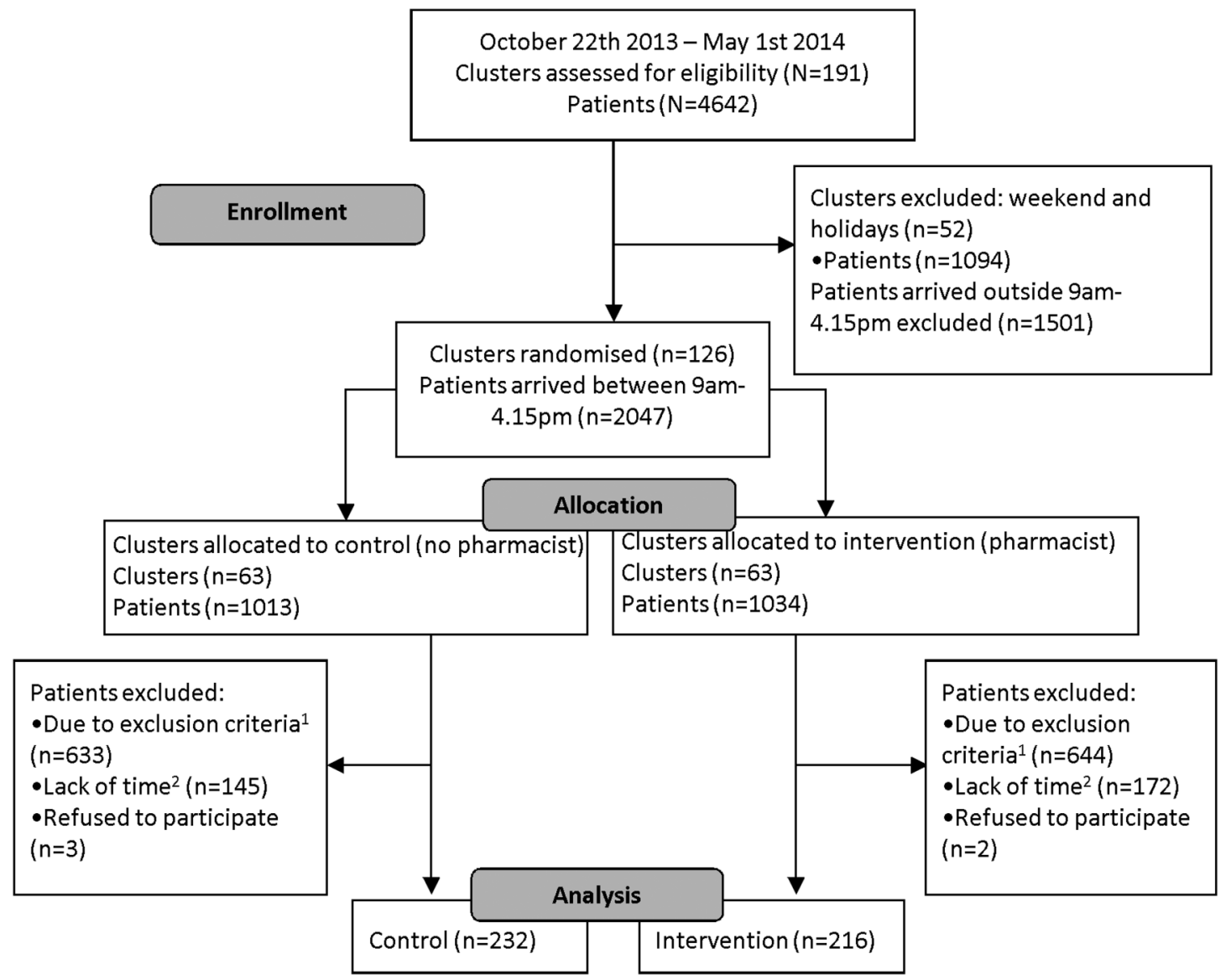

Figure 2 Flow of both clusters and patients due to the cluster randomised design. The top part illustrates the exclusion of clusters and patients outside the intervention shifts. The bottom part illustrates the exclusion of patients due to exclusion criteria and lack of time. ${ }^{1}$ Exclusion criteria: triage red: $3.3 \%$; $<18$ years, $2.0 \%$; $<4$ medications, $45.7 \%$; terminal or detoxified, $1.2 \%$; unable to give informed consent, $11.0 \%$; referred for acute outpatient clinic, 2.6\%; other reason-for example, referred to emergency department or medication already approved by physician, $12.7 \%$. ${ }^{2}$ Lack of time covers two scenarios: (1) physician interviewed patients before informed consent was obtained; (2) triage was not finish before 4.30pm (16:30), so there was not enough time for informed consent to be obtained and possibly intervention to be carried out before end of shift.

Continuous non-time variables in each group were summarised using means, and differences between groups were tested with two-sided $\mathrm{t}$ tests. ICC was calculated using long one-way analysis of variance by using the loneway command in Stata.

\section{RESULTS}

\section{Participant flow}

The study period consisted of 126 weekday shifts (09:00$16: 15)$, with 63 clusters in each study group. After exclusion of patients who did not meet the inclusion criteria, 216 and 232 were included and analysed in the intervention and control clusters, respectively (figure 2).

\section{Baseline data}

Baseline characteristics of patients in the two study groups were similar (table 2).

Overall, medical patients accounted for $71.9 \%$ of patients included. Most were hospitalised-that is, admitted to a hospital department $(82.8 \%)$.

\section{Outcomes and estimations}

Overall, there was no difference in patient LOS. Mean LOS was 339 (95\% CI 322 to 357 ) $\mathrm{min}$ in the control group. 342 (95\% CI 323 to 362) $\mathrm{min}$ in the intervention group and

The unadjusted regression analysis of LOS showed that LOS was on average $0.9 \%$ ( $3.2 \mathrm{~min})$ longer for patients in the intervention group compared with the control group $(p=0.83)$ (table 3 ).

Adjusting for medical specialty increased the difference in median LOS to $1.9 \%$, which was not a statistically significant difference. Neither did stratification for discharge or hospitalisation reveal any significant difference in LOS between the study groups (table 4).

Table 1 Secondary outcomes

\begin{tabular}{|c|c|}
\hline Outcome & Source of data collection \\
\hline \multicolumn{2}{|l|}{ All patients } \\
\hline $\begin{array}{l}\text { Time from arrival to a treatment plan (TATP) is } \\
\text { documented in the EMR }\end{array}$ & $\begin{array}{l}\text { Data registered in the EMR by } \\
\text { secretaries/physicians }\end{array}$ \\
\hline \multicolumn{2}{|l|}{ Physician time spent on medication topics } \\
\hline $\begin{array}{l}\text { Physician time spent on each patient including } \\
\text { examination, medication topics and } \\
\text { documentation }\end{array}$ & $\begin{array}{l}\text { Self-reporting on a standard } \\
\text { form }\end{array}$ \\
\hline $\begin{array}{l}\text { Number of medications per patient } \\
\text { Intervention group only }\end{array}$ & Data in EMR and EMM \\
\hline Pharmacist time spent on intervention & $\begin{array}{l}\text { Self-reporting on a standard } \\
\text { form }\end{array}$ \\
\hline
\end{tabular}

Lind KB, et al. Eur J Hosp Pharm 2016;23:171-176. doi:10.1136/ejhpharm-2015-000767 
Table 2 Baseline characteristics

\begin{tabular}{|c|c|c|c|}
\hline Characteristic & Control & Intervention & $\begin{array}{l}\mathrm{p} \\
\text { Value }\end{array}$ \\
\hline \multicolumn{4}{|l|}{ Patient level } \\
\hline Patients, n (\%) & $232(51.8)$ & $216(48.2)$ & - \\
\hline Age, mean (SD) & $69.8(12.7)$ & $70.9(13.8)$ & 0.36 \\
\hline Gender, men (\%) & $107(46.1)$ & $109(50.5)$ & 0.36 \\
\hline Patient category, medical (\%) & $161(69.4)$ & $161(74.5)$ & 0.23 \\
\hline Radiological imaging, n (\%) & $133(57.3)$ & $131(60.6)$ & 0.48 \\
\hline Triage & - & - & 0.60 \\
\hline Level 2, n (\%) & $30(12.9)$ & $33(15.3)$ & - \\
\hline Level 3, n (\%) & $148(63.8)$ & $122(56.5)$ & - \\
\hline Level 4, n (\%) & $54(23.3)$ & $61(28.2)$ & - \\
\hline Hospitalised, n (\%) & $188(81.0)$ & $183(84.7)$ & 0.30 \\
\hline Discharged to GP, n (\%) & $44(19.0)$ & $33(15.3)$ & 0.30 \\
\hline \multicolumn{4}{|l|}{ Cluster level } \\
\hline Number of days, $n(\%)$ & $63(50.0)$ & $63(50.0)$ & - \\
\hline $\begin{array}{l}\text { Patients admitted to AAU per } 24 \mathrm{~h} \text {, } \\
\text { mean }\end{array}$ & 28.0 & 28.3 & - \\
\hline $\begin{array}{l}\text { Patients admitted to AAU 09:00-16:15, } \\
\text { mean }\end{array}$ & 16.1 & 16.4 & - \\
\hline Physicians involved, $n^{*}$ & 75 & 81 & \\
\hline
\end{tabular}

Similarly, there was no significant difference in TATP between the study groups (table 3). The mean TATP was 205 (95\% CI 192 to 219) $\mathrm{min}$ in the intervention group and 193 (95\% CI 181 to 205) $\mathrm{min}$ in the control group.

The mean self-reported physician time spent on medication topics was $4.3(95 \%$ CI 3.7 to 5.0$) \mathrm{min}$ in the intervention group and 7.5 (95\% CI 6.6 to 8.5 ) $\mathrm{min}$ in the control group, corresponding to an overall reduction of $43.0 \%$ (95\% CI $30.9 \%$ to $53.0 \% ; \mathrm{p}<0.01)$.

The mean difference in self-reported physician time spent on each patient was insignificantly lower (-4.2\% (95\% CI $-15.0 \%$ to $8.0 \%, \mathrm{p}=0.58)$ ) when the intervention group $(52.2$ (95\% CI 48.8 to 57.2$) \mathrm{min}$ ) was compared with the control group (54.5 (95\% CI 50.4 to 59.0 ) $\mathrm{min}$ ).

The number of medications documented in the EMR was significantly $(p=0.002)$ higher among patients whose medication history was recorded by a CP $(10.0$ (95\% CI 9.5 to 10.6)

Table 3 Primary outcome

\begin{tabular}{|c|c|c|c|}
\hline & $\begin{array}{l}\text { Difference* } \\
\%(95 \% \mathrm{Cl})\end{array}$ & $\begin{array}{l}\text { Difference* } \\
\text { Minutes }(95 \% \mathrm{Cl})\end{array}$ & $\mathrm{p}$ Value \\
\hline \multicolumn{4}{|l|}{ Unadjusted } \\
\hline LOS & $0.9(-7.4$ to 10.1$)$ & $3.2(-25.2$ to 34.2$)$ & 0.83 \\
\hline TATP & $5.5(-5.1$ to 17.3$)$ & $10.7(-9.8$ to 33.5$)$ & 0.32 \\
\hline \multicolumn{4}{|c|}{ Adjusted for specialty } \\
\hline LOS & $1.9(-6.6$ to 11.0$)$ & & 0.67 \\
\hline Medical & & $6.0(-21.1$ to 35.5$)$ & \\
\hline Surgical & & 7.4 (-24.8 to 42.5$)$ & \\
\hline TATP & $4.3(-5.9$ to 15.5$)$ & & 0.42 \\
\hline Medical & & $8.8(-12.1$ to 31.9$)$ & \\
\hline Surgical & & $7.2(-10.0$ to 26.2$)$ & \\
\hline
\end{tabular}

medications) than among patients whose medication history was conducted by a physician $\left(\begin{array}{llllll}8.8 & (95 \% & \text { CI } & 8.3 & \text { to } & 9.4)\end{array}\right.$ medications).

CPs spent on average 33.6 (95\% CI 31.9 to 35.5) min per patient. Disregarding time spent on inserting the note into the EMR, CPs on average spent 24.4 (95\% CI 23.2 to 25.7 ) $\mathrm{min}$ per patient; 11.1 (95\% CI 10.4 to 11.8$)$ min was spent on obtaining the medication history.

\section{DISCUSSION}

Our findings did not indicate any statistically significant differences in LOS or TATP between the study groups regardless of adjustment of data for specialty and hospitalisation/discharge status. To the best of our knowledge, no other studies have investigated the consequences of CP interventions in an AAU or an ED on time variables such as LOS or TATP.

Various factors have been found to be significantly associated with prolonged LOS in EDs-for example, arrival mode, acuity level, receipt of medication, and ethnicity. ${ }^{15-18}$ The most commonly reported factors are blood tests and advanced radiological imaging. ${ }^{16-18}$ Almost all patients admitted to the AAU have a blood test. In our cohort, 59\% had at least one kind of radiological imaging during the AAU visit. It is probable that many of these factors associated with increased ED LOS are also associated with prolonged LOS in the AAU.

The association between the predictive factors mentioned above and prolonged ED LOS is stronger for discharged patients than for hospitalised patients. ${ }^{16}{ }^{18}$ ED LOS of hospitalised patients is strongly associated with in-hospital factors such as hospital occupancy. ${ }^{3}$ In contrast with previous results, ${ }^{15}{ }^{16}$ we found that LOS was shorter for admitted than discharged patients. This finding may be due to a different distribution of triage levels among admitted versus discharged patients, as $40 \%$ of discharged patients were assigned to the lowest triage level compared with $23 \%$ of admitted patients. Other possible explanations may be logistical problems related to discharge-for example, coordination of transportation. Finally, patients being transferred to a hospital ward may be less likely to have their treatment completed in the AAU. Although, in the literature, consultations ${ }^{15}$ and specialist advice ${ }^{17}$ have been associated with longer LOS, our findings suggest that this was not the case for the CP intervention in our study.

We found that physicians spent 7.5 min on medication topics in the control group. A Danish observational study of a CP intervention in an AAU used stopwatches to measure time variables and found that physicians on average spent $6.6 \mathrm{~min}$ on obtaining medication history and entering the prescriptions into the EMR. ${ }^{9}$ We therefore assume that our finding of a $43 \%$ reduction in time spent on medication topics is probably trustworthy despite being based on self-reported measures.

We found a significant difference in the number of medications per patient when comparing the groups: 10.0 (intervention) vs 8.8 (control). This can probably be explained by omission of drugs, which is the most commonly reported error $^{4-6} 8 \quad 1920$ when a standard medication history conducted by a physician is compared with a comprehensive medication history conducted by a CP. Similarly, it has been reported that CPs documented 614 and physicians documented 556 medications in the medication history for the same 55 patients. ${ }^{21}$ In our study, physicians and CPs did not conduct medication histories on the same patients, preventing such a comparison. However, considering the randomised study design and the equal distribution of baseline data between groups, it could be 
Table 4 Unadjusted LOS stratified by hospitalised/discharged status

\begin{tabular}{|c|c|c|c|c|c|}
\hline & $\begin{array}{l}\text { Control } \\
\text { Minutes (mean) }\end{array}$ & $\begin{array}{l}\text { Intervention Minutes } \\
\text { (mean) }\end{array}$ & $\begin{array}{l}\text { Difference* } \\
\%(95 \% \mathrm{Cl})\end{array}$ & $\begin{array}{l}\text { Difference*, Minutes } \\
(95 \% \mathrm{Cl})\end{array}$ & $p$ Value \\
\hline Hospitalised & 328 & 339 & $3.4(-5.9$ to 13.6$)$ & $11.1(-19.5$ to 44.7$)$ & 0.49 \\
\hline Discharged & 390 & 360 & $-7.8(-22.6$ to 9.9$)$ & $-30.3(-88.3$ to 38.8$)$ & 0.36 \\
\hline
\end{tabular}

expected that the average number of medications per patient would be the same in the two groups.

The CPs took on average 11.1 min to conduct each medication history in our study. In line with existing literature, ${ }^{5} 21$ this finding indicates that completion of a comprehensive medication history is time-consuming. In a recent study, pharmacy technicians on average took 44 min to obtain a medication history and write notes in the EMR. ${ }^{22}$ Other studies have reported about $15 \min ^{521}$ and 9-30 min. ${ }^{6}$

We found that CPs, in total, spent $33.5 \mathrm{~min}$ per patient. This is less than the $44 \mathrm{~min}$ found in a comparable Danish study ${ }^{12}$ and longer than the $21.2 \mathrm{~min}$ found by Gleason et al. ${ }^{20} \mathrm{In}$ general, it is difficult to compare results of time expenditures, since most studies do not state exactly what components were actually covered. In Denmark, clinicians and CPs have access to a number of medication sources-for example, EMR, EMM and the shared medication record, ${ }^{11}$ where the prescribed medicine purchased by patients at community pharmacies can be viewed directly. Access to and use of these advanced electronic systems contributes to the overall quality of drug treatment; however, it is also associated with increased time spent and might explain why the CP intervention in our study exceeded findings from abroad. ${ }^{20}$

The difference in time consumption between CPs and physicians was notable. However, a similar difference was found between pharmacy technicians and physicians. ${ }^{22}$ CPs and pharmacy technicians focus on the patient's medication only, in contrast with physicians who also have to address all aspects of the medical examination-often, within a limited time span. These conditions probably explain the marked difference in time consumption between the professions.

The need for support and advice on medication topics was investigated in a large Danish questionnaire survey: $73 \%$ of all physicians and $92 \%$ of junior physicians agreed that there is a need for external medication counselling. ${ }^{23}$

This need, along with our finding of saved physician time, suggests that $\mathrm{CP}$ intervention involving both medication history and medication review would be useful in AAU settings, despite the finding of no overall impact on LOS. Further investigations are required to confirm this hypothesis. In addition, the effect of CP interventions on quality and economic issues is needed.

\section{Limitations of the study}

First, several circumstances reduce the generalisability: the study was carried out in one hospital only and the number of excluded patients was large because of wide exclusion criteria.

Second, some of the time measures were based on selfreporting of time consumption, which increases the likelihood of these results being inaccurate. In addition, we did not investigate how the physicians spent their saved minutes.

Third, physicians may have used some knowledge gained from the intervention on patients in the control group. However, the limited time spent on medication topics means that it is unlikely that the time-related end points will have been influenced by this.

Fourth, LOS in AAUs was influenced by a number of factors, and may therefore not be the most appropriate measure of a single and brief intervention such as ours, bearing in mind that the physicians on average spent $7.5 \mathrm{~min}$ on medication topics. An obvious measure of time-saving interventions would be use of stopwatches by an external observer. In addition, TATP may be a more appropriate measure; however, it would require far more valid and reliable registration methods than currently available in our AAU.

Fifth, documenting a treatment plan in the EMR was a new task for the physicians when the study began. During the study, increased attention was paid to this task, potentially leading to a systematic change in the timing of the documentation; however, this shortcoming applies to both study groups.

Finally, we stopped the trial before reaching the required sample size because of substantial organisational changes in terms of different location, workflow and standards of hospitalisation, which would have had a great impact on LOS and severely influenced the completion of the intervention. We managed to include $51.3 \%$ of the required study population and therefore cannot rule out that the intervention would have shown a significant impact on the primary end point. On the other hand, the findings did not indicate any tendencies towards a reduction in LOS or TATP after inclusion of 448 patients. In addition, the log-transformed ICC in our study population was 0.04 , which is less than the estimated ICC $(0.05)$ used in the sample-size calculation. This indicates a higher variability between LOS for patients in the same cluster-that is, admitted during the same day shift-than LOS for patients in different clusters. Use of 0.04 instead of 0.05 would have led to a requirement for a slightly smaller study sample. Consequently, the power of the presented result is estimated to be 0.59 when the following data sample size is applied: 232 and 216 patients, ICC 0.04, and cluster size 3.56 patients/day.

\section{What this paper adds}

What is already known on this subject?

- Many different factors influence length of stay in admission units.

- Several studies have shown that clinical pharmacists obtain more comprehensive medication histories than physicians.

What this study adds?

- This study indicates that length of stay in an admission unit is not affected by a clinical pharmacist (CP) intervention.

- The CP intervention replaces most of the physician's tasks regarding medications in the acute admission unit. 


\section{CONCLUSION}

This study indicates that adding a CP to our AAU team did not affect LOS or TATP. Neither was the time spent by physicians on each patient affected. On the other hand, physicians spent $43 \%(\mathrm{p}<0.001)$ less time on medication-related topics when the patients' medications were managed by the CP. In summary, the flow in the AAU was not affected by the CP intervention, but physicians saved time on medication-related topics, which could potentially be used for other tasks.

Acknowledgements We would like to thank the Department of Biostatistics at Aarhus University and physicians at the AAU at Randers Regional Hospital. Special thanks go to the clinical pharmacist, Anita Buch Grann Press.

Contributors $\mathrm{KBL}, \mathrm{CAS}, \mathrm{SAS}$ and $\mathrm{ML}$ were responsible for the planning and design of the study. KBL and CAS were responsible for data collection and analysis. KBL and CAS wrote the first draft; SAS and ML provided critical revision; all authors read and approved the final manuscript.

Funding This study was funded by the Research Center for Emergency Medicine at Aarhus University Hospital, Denmark, The Hospital Pharmacy of Aarhus and Randers Regional Hospital, Denmark.

Competing interests None declared.

Patient consent Obtained.

Ethics approval The regional ethics committee waived the need for approval.

Provenance and peer review Not commissioned; externally peer reviewed.

\section{REFERENCES}

1 Sun BC, Hsia RY, Weiss RE, et al. Effect of emergency department crowding on outcomes of admitted patients. Ann Emerg Med 2013;61:605-11.e6.

2 McCarthy ML, Zeger SL, Ding R, et al. Crowding delays treatment and lengthens emergency department length of stay, even among high-acuity patients. Ann Emerg Med 2009;54:492-503.e4.

3 Forster AJ, Stiell I, Wells G, et al. The effect of hospital occupancy on emergency department length of stay and patient disposition. Acad Emerg Med 2003; 10:127-33.

4 Basey AJ, Krska J, Kennedy TD, et al. Prescribing errors on admission to hospital and their potential impact: a mixed-methods study. BMJ Qual Saf 2014;23:17-25.

5 De Winter S, Spriet I, Indevuyst C, et al. Pharmacist- versus physician-acquired medication history: a prospective study at the emergency department. Qual Saf Health Care 2010;19:371-5.
6 Tam VC, Knowles SR, Cornish PL, et al. Frequency, type and clinical importance of medication history errors at admission to hospital: a systematic review. CMAJ 2005;173:510-15.

7 Kwan JL, Lo L, Sampson M, et al. Medication reconciliation during transitions of care as a patient safety strategy: a systematic review. Ann Intern Med 2013;158(5 Pt 2):397-403.

8 Galvin M, Jago-Byrne MC, Fitzsimons M, et al. Clinical pharmacist's contribution to medication reconciliation on admission to hospital in Ireland. Int I Clin Pharm 2013;35:14-21

9 Ghazanfar MN, Honore PH, Nielsen TR, et al. Hospital admission interviews are time-consuming with several interruptions. Dan Med J 2012;59:A4534.

10 DEPT Triage System. http://deptriage.dk/ (accessed Oct 2015).

11 Shared Medication Record. http://www.ssi.dk/English/HealthdataandICT/The\% 20National\%20eHealth\%20Authority/FMK.aspx (accessed 10 Dec 2015).

12 Nielsen TR, Andersen SE, Rasmussen $M$, et al. Clinical pharmacist service in the acute ward. Int J Clin Pharm 2013;35:1137-51.

13 Li J, Caviness AC, Patel B. Effect of a triage team on length of stay in a pediatric emergency department. Pediatr Emerg Care 2011;27:687-92.

14 van Tulder $R$, Roth $D$, Weiser $C$, et al. An electrocardiogram technician improves in-hospital first medical contact-to-electrocardiogram times: a cluster randomized controlled interventional trial. Am J Emerg Med 2012;30:1729-36.

15 Brick $C$, Lowes J, Lovstrom $L$, et al. The impact of consultation on length of stay in tertiary care emergency departments. Emerg Med J 2014;31:134-8.

16 Gardner RL, Sarkar U, Maselli JH, et al. Factors associated with longer ED lengths of stay. Am J Emerg Med 2007;25:643-50.

17 Casalino E, Wargon M, Peroziello $A$, et al. Predictive factors for longer length of stay in an emergency department: a prospective multicentre study evaluating the impact of age, patient's clinical acuity and complexity, and care pathways. Emerg Med J 2014;31:361-8

18 Kocher KE, Meurer WJ, Desmond JS, et al. Effect of testing and treatment on emergency department length of stay using a national database. Acad Emerg Med 2012;19:525-34.

19 Vasileff HM, Whitten LE, Pink JA, et al. The effect on medication errors of pharmacists charting medication in an emergency department. Pharm World Sci 2009:31:373-9.

20 Gleason KM, McDaniel MR, Feinglass J, et al. Results of the Medications at Transitions and Clinical Handoffs (MATCH) study: an analysis of medication reconciliation errors and risk factors at hospital admission. J Gen Intern Med 2010;25:441-7

21 Reeder TA, Mutnick A. Pharmacist- versus physician-obtained medication histories. Am J Health Syst Pharm 2008;65:857-60.

22 Henriksen JP, Noerregaard S, Buck TC, et al. Medication histories by pharmacy technicians and physicians in an emergency department. Int J Clin Pharm 2015:37:1121-7.

23 Bonnerup DK, Lisby M, Eskildsen AG, et al. Medication counselling: physicians' perspective. Basic Clin Pharmacol Toxicol 2013;113:425-30. 